\title{
Pathological complete response in a case of HER2-positive gastric cancer with peritoneal dissemination treated with trastuzumab in combination with chemotherapy
}

\author{
Yuki Kiyozumi • Masayuki Watanabe - Masaaki Iwatsuki • \\ Takatsugu Ishimoto $\cdot$ Shiro Iwagami $\cdot$ Yoshifumi Baba \\ Yuka Tamaoki $\cdot$ Kenichi Iyama $\cdot$ Hideo Baba
}

Received: 14 July 2012/ Accepted: 8 October 2012/Published online: 9 November 2012

(C) The Japan Society of Clinical Oncology 2012

\begin{abstract}
Trastuzumab in combination with cisplatin and capecitabine could be a new standard treatment for patients with HER2-positive advanced gastric cancer. We herein report a case of advanced gastric cancer with peritoneal dissemination that was successfully treated with trastuzumab combined with cisplatin and capecitabine followed by gastrectomy. A 49-year-old man complained of epigastric pain and was diagnosed with advanced gastric cancer. A staging laparoscopy revealed peritoneal dissemination that was overexpressing HER2 protein. After five courses of chemotherapy comprising trastuzumab, cisplatin and capecitabine, laparoscopic restaging was performed. The disseminated nodules had disappeared, and biopsy of the scar of peritoneal seeding and cytology of the peritoneal lavage fluid both found no malignant cells. Therefore, total gastrectomy with D2 lymph node dissection was performed. Pathologically, there were no residual tumor cells in the resected stomach or peritoneal wall. Trastuzumab in combination with chemotherapy may have curative potential for peritoneal dissemination from HER2positive gastric cancer.
\end{abstract}

Keywords Gastric cancer - Trastuzumab - Pathological complete response

\footnotetext{
Y. Kiyozumi · M. Watanabe - M. Iwatsuki - T. Ishimoto ·

S. Iwagami · Y. Baba · Y. Tamaoki · H. Baba $(\bowtie)$

Department of Gastroenterological Surgery, Graduate School of

Medical Sciences, Kumamoto University,

1-1-1 Honjo, Kumamoto 860-8556, Japan

e-mail: hdobaba@kumamoto-u.ac.jp

K. Iyama

Department of Pathology, Graduate School of Medical Sciences,

Kumamoto University, 1-1-1 Honjo,

Kumamoto 860-8556, Japan
}

\section{Introduction}

Gastric cancer is a common malignancy and is associated with a high global mortality rate. In 2008, approximately 988,000 new cases were diagnosed, and 736,000 individuals died of gastric cancer worldwide. In particular, gastric cancer is the second leading cause of cancer-related death in East Asia, including Japan, Korea, and China [1]. Peritoneal dissemination is the most common pattern of recurrence or metastasis in gastric cancer [2], and is responsible for approximately $60 \%$ of all deaths from gastric cancer. Although a meta-analysis of phase II and III randomized gastric cancer trials showed that combination chemotherapy results in substantially improved overall survival [3], patients with peritoneal dissemination have very poor prognoses, with a median survival of 3-6 months $[4,5]$.

Recently, new molecular target therapy agents and combination chemotherapy regimens that are effective against gastric cancer have been developed. Trastuzumab, a monoclonal antibody that targets HER2, induces antibody-dependent cellular cytotoxicity and inhibits HER2mediated signaling, resulting in tumor cell proliferation, apoptosis, adhesion, migration, and differentiation [6]. In HER2-positive breast cancer, trastuzumab has shown a survival advantage in early and metastatic disease, and is now the standard of care for breast cancer [7, 8]. Recently, the Trastuzumab for Gastric Cancer (ToGA) trial revealed that trastuzumab in combination with chemotherapy could be a new standard treatment option for patients with HER2positive advanced gastric cancer [9].

We herein present a case of a 49-year-old man with advanced gastric cancer with HER2-overexpressing peritoneal dissemination that was successfully treated with trastuzumab in tandem with current combination 
chemotherapy. The peritoneal dissemination completely disappeared, and the patient then underwent total gastrectomy. To the best of our knowledge, there have been no previous reports of cases that underwent curative-intent surgery after trastuzumab in combination with chemotherapy for peritoneal dissemination.

\section{Case report}

A 49-year-old Japanese male complaining of epigastric pain underwent an esophagogastroduodenoscopy (EGD), which revealed an ulcerative lesion on the lesser curvature of the gastric angle (Fig. 1a). Biopsy of the gastric ulcer demonstrated poorly differentiated adenocarcinoma (Fig. 1b). The tumor was strongly positive for HER2 immunohistochemical (IHC) staining (Fig. 1c), and an abdominal ultrasound and computed tomography (CT) scan showed lymph node metastases; however, no obvious peritoneal dissemination was observed. A staging laparoscopy revealed peritoneal dissemination, and both the biopsy of the peritoneal tumor and peritoneal lavage cytology demonstrated adenocarcinoma, which was also positive for HER2 immunostaining (Fig. 2a, b). The patient received a trastuzumab-based regime, as follows: $8 \mathrm{mg} / \mathrm{kg}$ intravenous (i.v.) trastuzumab as the loading dose and $6 \mathrm{mg} / \mathrm{kg}$ as the maintenance dose on day $1,80 \mathrm{mg} / \mathrm{m}^{2}$ i.v. cisplatin on day 1 , and $2,000 \mathrm{mg} / \mathrm{m}^{2} /$ day capecitabine on day 1 to day 14, every 3 weeks. He received five cycles of chemotherapy without suffering from any severe toxicities except for grade 3 neutropenia. Post-treatment imaging studies were performed. EGD showed significant shrinkage of the tumor, and a CT scan revealed the disappearance of metastatic lymph nodes. To evaluate the peritoneal dissemination, staging laparoscopy was performed again. The peritoneal tumors had completely disappeared (Fig. 2c), and biopsy of the scar of the peritoneal tumor and peritoneal lavage cytology both found no malignant cells. Therefore, total gastrectomy with D2 lymph node dissection, reconstructed with Roux-en-Y esophagojejunostomy, was performed (Fig. 3a, b). Pathological examination of the surgical specimen revealed no residual tumor cells
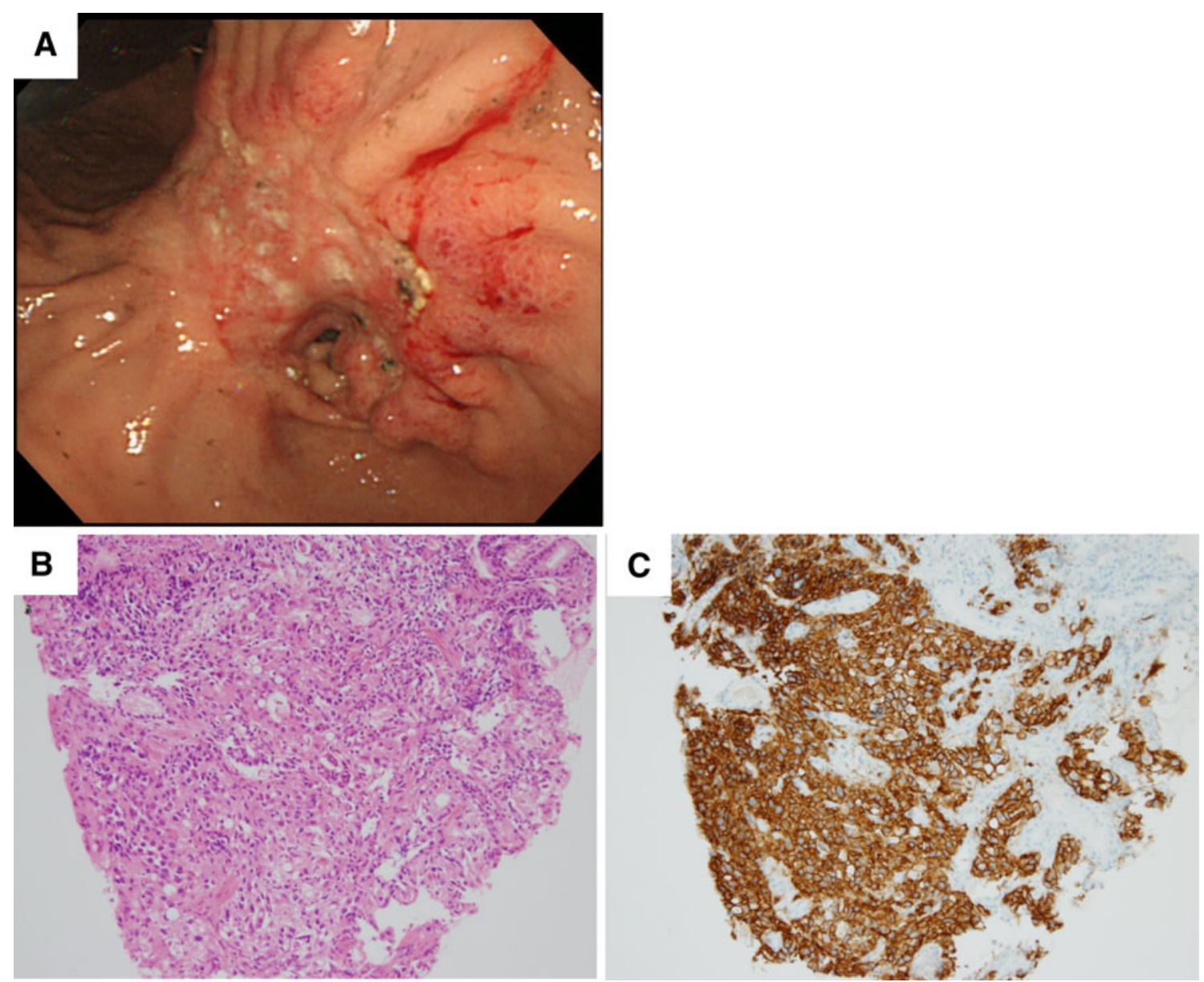

Fig. 1 a EGD revealed an ulcerous lesion on the lesser curvature of the gastric angle. b Biopsy of the gastric ulcer demonstrated poorly differentiated adenocarcinoma $(\times 40)$. $\mathbf{c}$ The tumor was strongly positive for HER-2 IHC staining $(\times 40)$ 

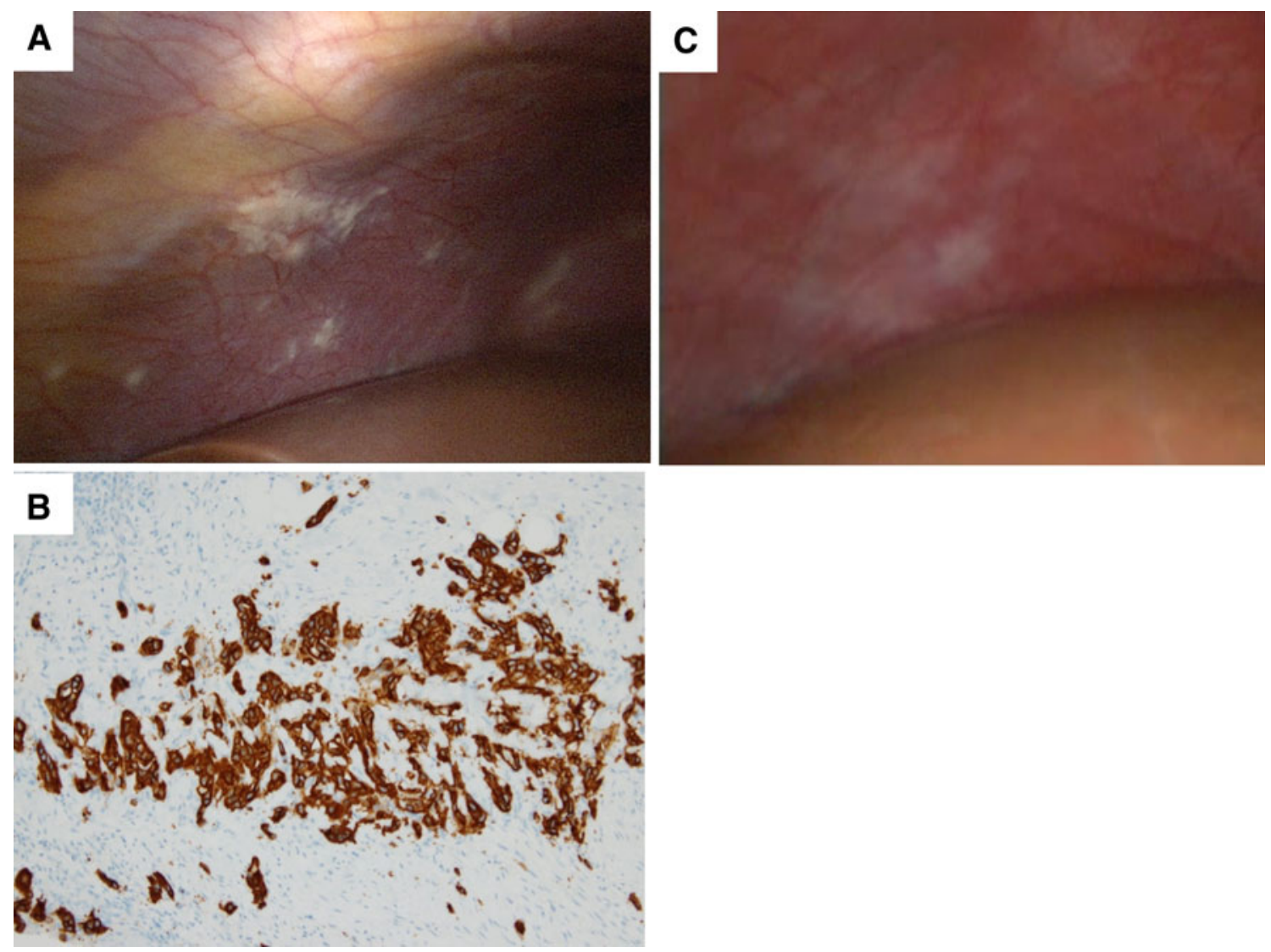

Fig. 2 a Staging laparoscopy revealed peritoneal dissemination. b Biopsy of the peritoneal tumor demonstrated adenocarcinoma that was positive for HER-2 immunostaining $(\times 40)$. c The peritoneal

(Fig. 3c). No tumor tissue was identified in 39 retrieved lymph nodes. He recovered uneventfully after surgery, and was discharged after 15 postoperative days.

\section{Discussion}

We herein present a case of gastric cancer with peritoneal dissemination that was successfully treated with trastuzumab plus capecitabine and cisplatin. A pathological complete response was achieved in the resected specimens, which included the primary lesion, lymph nodes, and peritoneal dissemination. There are two case reports suggesting the advantages of combined trastuzumab and chemotherapy in preoperative settings for HER-2-positive locally advanced gastric cancer [10, 11]. Wang et al. [11] and Sbitti et al. [10] reported cases of complete pathological response after neoadjuvant chemotherapy with a trastuzumab-containing regimen for resectable locally advanced gastric cancer overexpressing HER2. However, to our knowledge, there are no previous reports that have demonstrated the complete response of peritoneal tumors completely disappeared, leaving scar tissue that was pathologically free from cancer cells

dissemination to a trastuzumab-based regimen, resulting in curative gastrectomy.

Peritoneal dissemination is the most common type of recurrence or metastasis in gastric cancer, and it leads to a poor prognosis [2]. Therefore, it is critical to treat peritoneal dissemination to improve the prognosis of gastric cancer. However, it is often difficult to detect peritoneal dissemination by conventional imaging examination in clinical practice. Staging laparoscopy is very useful for identifying intra-abdominal tumor deposits on the peritoneal surface that are not detectable by preoperative noninvasive imaging. Nakagawa et al. [12] suggested that staging laparoscopy plays two critical roles in cases of advanced gastric cancer; the avoidance of an unnecessary laparotomy in patients with incurable metastatic diseases, and the exact staging of patients for appropriate preoperative treatment. Our indication for staging laparoscopy is large macroscopic type 3 or 4 gastric cancer with serosal invasion without hematogenous or clinically evident peritoneal dissemination, as previously reported [13]. Initially, staging laparoscopy confirmed that our patient had peritoneal dissemination even though peritoneal dissemination was not detected by CT. Using specimens obtained at 


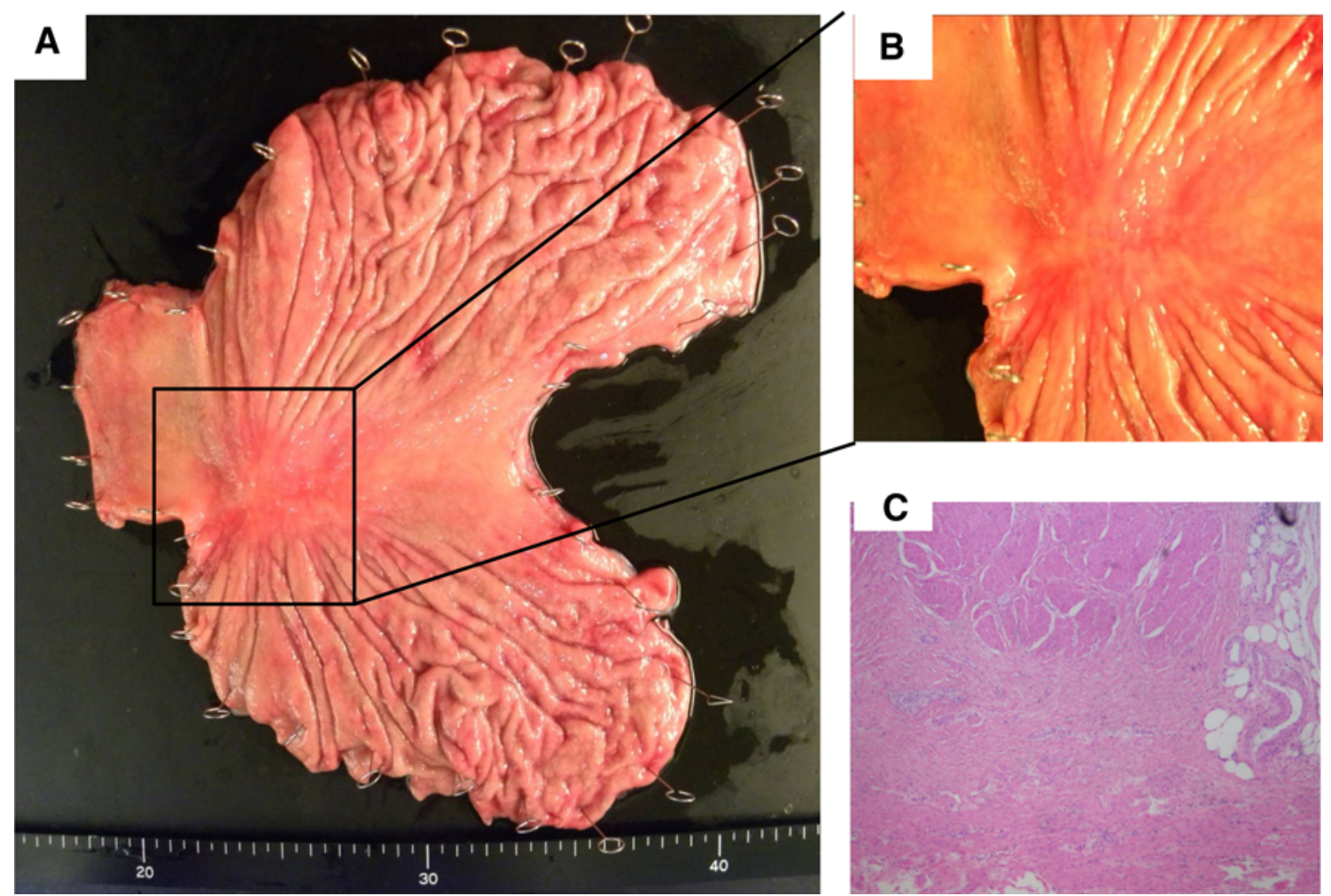

Fig. 3 a, b Macroscopic findings in the resected stomach. Significant shrinkage of the tumor was observed. c Microscopically, no viable cancer cells were observed $(\times 100)$

laparoscopy, the pathological diagnosis of peritoneal dissemination overexpressing HER2 was confirmed by IHC.

The ToGA trial was the first randomized phase III trial to show that trastuzumab plus chemotherapy is superior to chemotherapy alone for HER2-positive advanced or metastatic gastric cancer [9]. Trastuzumab plus 5-fluorouracil or capecitabine and cisplatin has become the standard treatment for patients with HER2-positive metastatic gastric cancer. Either HER2 protein overexpression (as detected by IHC) or gene amplification (detected by fluorescent in situ hybridization, FISH) was observed in 4-28\% of gastric cancer cases $[14,15]$. The ToGA trial reported an overall HER2-positive rate of $21.3 \%$ [9]. In this trial, the overall tumor response rate was significantly improved in the trastuzumab plus chemotherapy group compared with the chemotherapy alone group (CR: 5 vs. $2 \%$, PR: 42 vs. $32 \%$ ), corresponding to a $26 \%$ reduction in the death rate [9]. Furthermore, exploratory analysis according to HER2 status suggested that overall survival was longer in patients with high expression of HER2 protein than in patients with low expression. However, there were no other promising factors beyond HER2 status in the exploratory analysis of the ToGA trial. Therefore, further analyses are required to elucidate other molecular biological mechanisms for predicting sensitivity to trastuzumab. In our case, it is possible that the addition of trastuzumab to 5-fluorouracil or capecitabine and cisplatin is more effective because both the primary tumor and disseminated tumor had strong HER2 expression.

Also, Cavanna et al. [16] investigated HER2 status in both primary and tumor cells in the cerebrospinal fluid using FISH, which allowed intrathecal administration of trastuzumab for leptomeningeal carcinomatosis of gastric cancer. Bozzetti et al. [17] reported that both IHC and FISH indicated a high concordance between HER2 expression on primary tumors and that on corresponding metastases in gastric cancer, suggesting that, in most cases, HER2 status remains unchanged during the metastatic process. In our patient, IHC gave a HER2-positive score of $3+$ for both the primary tumor and disseminated tumor.

However, there are a number of issues that need to be resolved in order to determine the exact HER2 status, because there are some differences between surgically resected specimens and biopsy fragments [18]. Therefore, the further development of methodology to assess HER2 status is required so that HER2 expression can be evaluated precisely by methods other than IHC and FISH.

To the best of our knowledge, this patient is the first reported case of curative gastrectomy after trastuzumab plus chemotherapy for peritoneal dissemination. Trastuzumab in combination with chemotherapy may have curative potential for peritoneal dissemination from HER2-positive gastric cancer. 
Acknowledgments We thank Mr. K. Miyake and Ms. Yokoyama for their excellent technical assistance. This work was supported in part by the following grants and foundations: a Japan Society for the Promotion of Science (JSPS) Grant-in-Aid for Scientific Research (grant number 23791550), Takeda Science Foundation 2010, Okukubo Memorial Fund for Medical Research in Kumamoto University School of Medicine 2010, Uehara Memorial Foundation 2010, and YOKOYAMA Foundation for Clinical Pharmacology 2011.

Conflict of interest We declare that we have no conflict of interest.

\section{References}

1. Ferlay HS, Shin H, Bray F, Forman D et al (2010) GLOBOCAN 2008: cancer incidence and mortality worldwide (IARC CancerBase no. 10). International Agency for Research on Cancer, Lyon

2. Boku T, Nakane Y, Minoura T et al (1990) Prognostic significance of serosal invasion and free intraperitoneal cancer cells in gastric cancer. Br J Surg 77:436-439

3. Wagner AD, Grothe W, Haerting J et al (2006) Chemotherapy in advanced gastric cancer: a systematic review and meta-analysis based on aggregate data. J Clin Oncol 24:2903-2909

4. Isobe Y, Nashimoto A, Akazawa K et al (2008) Gastric cancer treatment in Japan: 2008 annual report of the JGCA nationwide registry. Gastric Cancer 14:301-316

5. Koizumi W, Narahara H, Hara T et al (2008) S-1 plus cisplatin versus S-1 alone for first-line treatment of advanced gastric cancer (SPIRITS trial): a phase III trial. Lancet Oncol 9:215-221

6. Hudis CA (2007) Trastuzumab-mechanism of action and use in clinical practice. N Engl J Med 357:39-51

7. Slamon DJ, Leyland-Jones B, Shak S et al (2001) Use of chemotherapy plus a monoclonal antibody against HER2 for metastatic breast cancer that overexpresses HER2. N Engl J Med 344:783-792

8. Smith I, Procter M, Gelber RD et al (2007) 2-year follow-up of trastuzumab after adjuvant chemotherapy in HER2-positive breast cancer: a randomised controlled trial. Lancet 369:29-36
9. Bang YJ, Van Cutsem E, Feyereislova A et al (2010) Trastuzumab in combination with chemotherapy versus chemotherapy alone for treatment of HER2-positive advanced gastric or gastrooesophageal junction cancer (ToGA): a phase 3, open-label, randomised controlled trial. Lancet 376:687-697

10. Sbitti Y, Essaidi I, Debbagh A et al (2011) Is there any advantage to combined trastuzumab and chemotherapy in perioperative setting her 2neu positive localized gastric adenocarcinoma? World J Surg Oncol 9:112

11. Wang J, Saukel GW, Garberoglio CA et al (2012) Pathological complete response after neoadjuvant chemotherapy with trastuzumab-containing regimen in gastric cancer: a case report. J Hematol Oncol 3:31

12. Nakagawa S, Nashimoto A, Yabusaki H (2007) Role of staging laparoscopy with peritoneal lavage cytology in the treatment of locally advanced gastric cancer. Gastric Cancer 10:29-34

13. Leake PA, Cardoso R, Seevaratnam R et al (2012) A systematic review of the accuracy and indications for diagnostic laparoscopy prior to curative-intent resection of gastric cancer. Gastric Cancer 15(Suppl 1):38-47

14. Hofmann M, Stoss O, Shi D et al (2008) Assessment of a HER2 scoring system for gastric cancer: results from a validation study. Histopathology 52:797-805

15. Tanner M, Hollmen M, Junttila TT et al (2005) Amplification of HER-2 in gastric carcinoma: association with Topoisomerase IIalpha gene amplification, intestinal type, poor prognosis and sensitivity to trastuzumab. Ann Oncol 16:273-278

16. Cavanna L, Rocchi A, Gorgni S et al (2011) Cerebrospinal fluid cytology diagnosis of HER2-positive leptomeningeal carcinomatosis from HER2-positive metastatic gastric cancer: case report. J Clin Oncol 29:e367-e368

17. Bozzetti C, Negri FV, Lagrasta CA et al (2011) Comparison of HER2 status in primary and paired metastatic sites of gastric carcinoma. Br J Cancer 104:1372-1376

18. Shinozaki E, Yamamoto N, Chin K et al (2012) How many biopsy fragments will be necessary to assess HER2 status for gastric cancer? J Clin Oncol 30(Suppl 4):Abstr 40 